\title{
Optimization of Signal Detection in Scintillation Secondary Electron Detector for ESEM and SEM
}

\author{
Pavel Čudek ${ }^{1}$, Josef Jirák ${ }^{1,2}$, Vilém Neděla ${ }^{2}$ \\ 1. Department of Electrotechnology, Brno University of Technology, Brno, Czech Republic. \\ 2. Institute of Scientific Instruments of ASCR, Brno, Czech Republic.
}

Scintillation secondary electron detector for environmental scanning electron microscope (ESEM) that uses two pressure limiting apertures to decrease pressure in scintillator chamber to values which allow to connect voltage of $10 \mathrm{kV}$ to the scintillator while the water vapor pressure in the specimen chamber of the microscope reaches up to $1000 \mathrm{~Pa}$ was introduced in [1]. Furthermore the possibility of detection of secondary electrons with this detector, even at pressure of $0.01 \mathrm{~Pa}$ (vacuum conditions) in the specimen chamber was presented in [2]. In this detector, pictured on Fig.1, the space between two apertures A1 and A2 is vacuum pumped by a rotary pump and the scintillator chamber by a turbomolecular pump. Voltages connected to the grid, to electrodes E1 and E2 and to the electrostatic lens created by the apertures A1 and A2 produce an electrostatic field that attracts secondary electrons emitted from the specimen to the detector and allow them to pass to the scintillator chamber where they are accelerated to the scintillator.

For an effective detection of secondary electrons by this detector it is necessary to add voltage of $300 \mathrm{~V}$ or higher to the grid which is interconnected with the electrode E1 of the detector. It was experimentally proved [3] that optimal voltages on the electrodes of the detector are dependent on the pressure in the specimen chamber as well as on the pressure inside the detector. Pressure inside the detector is influenced by diameter of openings in the pressure limiting apertures A1 and A2. The highest signal level in the dependence on used diameter of openings in A1, A2 and in water vapor pressure up to 350 $\mathrm{Pa}$ in the specimen chamber can be obtained when setting voltage on the electrode system of the detector to UE1 $=300 \mathrm{~V}, \mathrm{UE} 2=400 \mathrm{~V}, \mathrm{UA} 1=550 \mathrm{~V}$ and UA1 $>1000 \mathrm{~V}$. However at these voltages on electrodes the signal level strongly decreases with the pressure increase in the specimen chamber, the detector can become unstable. Optimal voltages on the electrode system of the detector for water vapor pressure over $300 \mathrm{~Pa}$ in the specimen chamber are UE1 $=300 \mathrm{~V}, \mathrm{UE} 2=310-330 \mathrm{~V}, \mathrm{UA} 1=440 \mathrm{~V}$ and UA1 $>1000 \mathrm{~V}$. These voltages can be also used for pressure from $0.01 \mathrm{~Pa}$ in the specimen chamber (vacuum conditions). Model situation describing the signal level increase and decrease for the water vapor pressure range up to $1 \mathrm{kPa}$ and for different sets of voltages on electrodes of the detector is depicted on Fig. 2. This dependence is typically measured at constant voltage on dynodes of the detector photomultiplier. During standard sample observation the signal level increase and decrease can be compensated by changing the voltage on dynodes of the detector photomultiplier so that the sample pictures taken for example at the pressure of $0.01 \mathrm{~Pa}, 200 \mathrm{~Pa}$ and $500 \mathrm{~Pa}$ in the specimen chamber look very similarly. The second set of voltages on the detector electrodes (Fig. 2, red line) can be used for whole pressure range ideally. However in real conditions sometimes noise and discharges start appear at the sample observation caused by the pressure growth in the room between the apertures A1 and A2 of the detector. In this case it is necessary to decrease voltages on the electrode system of the detector to $\mathrm{UE} 1=100 \mathrm{~V}, \mathrm{UE} 2=110-130 \mathrm{~V}, \mathrm{UA} 1=200-300 \mathrm{~V}$ and $\mathrm{UA} 1 \geq 750 \mathrm{~V}$ in dependence on the pressure between apertures A1 and A2. Nevertheless the decrease of the voltage on the grid interconnected with electrode E1 leads to lowering of detection of secondary electrons emitted from the sample. Solution of this problem is connected with the use of smaller diameter of the opening in aperture A1, respectively 
with optimization of gas flow in the detector leading to the decrease of pressure in the space between A1 and $\mathrm{A} 2$.

Detection of secondary electrons in ESEM by the scintillation SE detector is influenced by voltage values connected to electrodes of the detector, used diameter of openings in pressure limiting apertures A1, A2 and by pressure values in individual chambers of the detector. This issue will be discussed in more detail in poster presentation.

\section{References:}

[1] J Jirák et al, Journal of Microscopy 239 (2010), p. 233. [2] J

Jirák et al, Microscopy and Microanalysis (2012) p. 1266. [3] J

Jirák et al, Microscopy and Microanalysis (2011) p. 922.

[4] This work was supported by the EU project No. CZ.1.07/2.3.00/20.0103 and by the Grant Agency of the Czech Republic: grant No. GA 14-22777S.

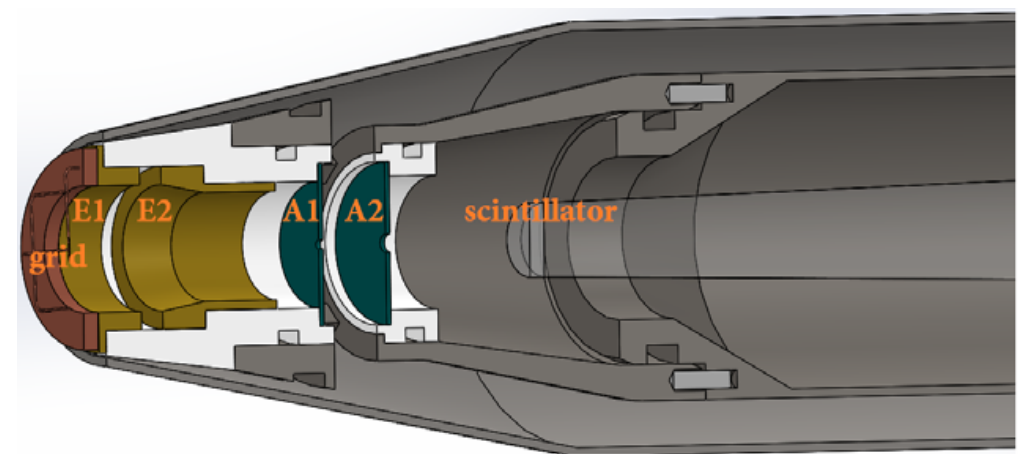

Figure 1. Sectional view of scintillation secondary electron detector for ESEM and SEM.

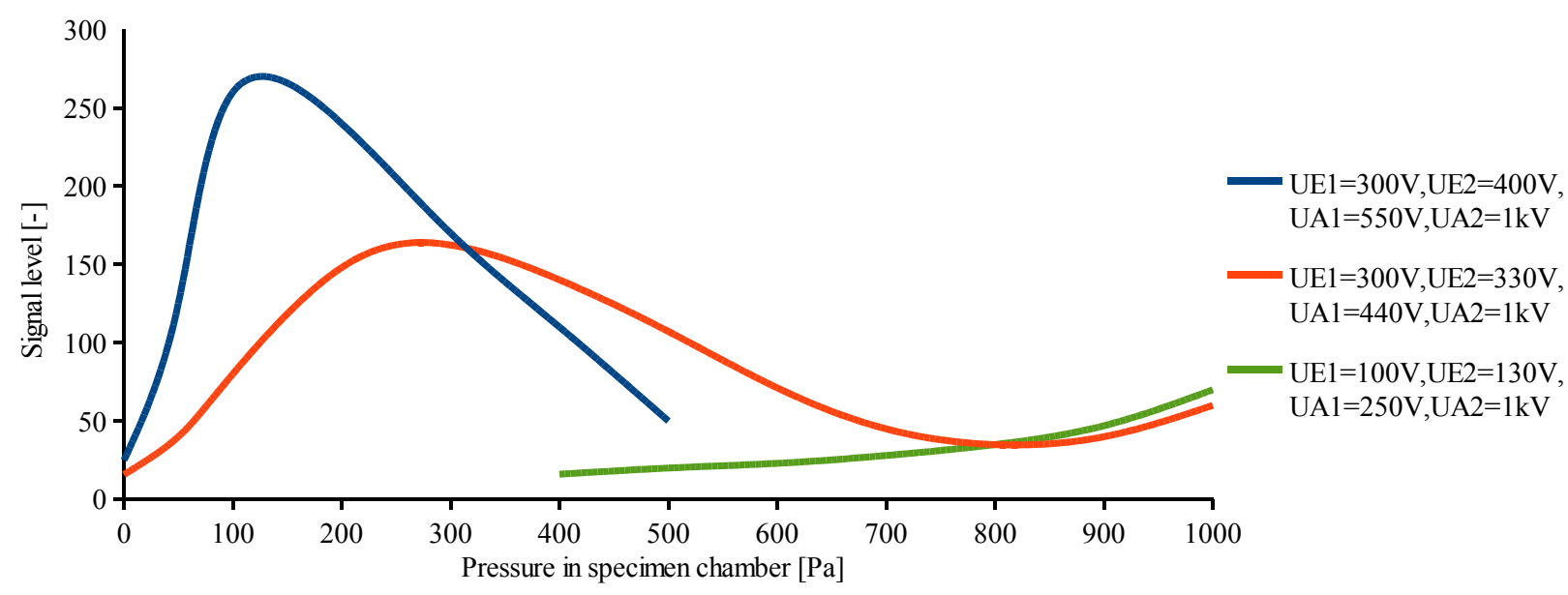

Figure 2. Dependences of signal level on water vapor pressure in specimen chamber for different sets of voltages on detector electrodes.

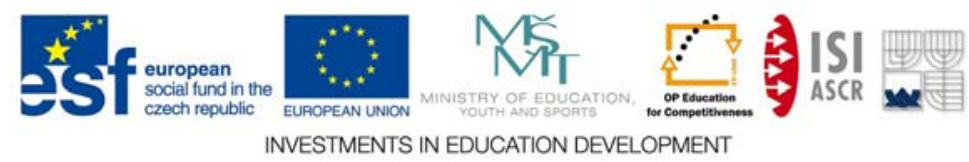

